\section{New set of eyes on the radio sky in the Southern Hemisphere}

Narrabri, New South Wales construction, Australia has entered the big league in world astronomy with the commissioning last week of the Australia Telescope.

The telescope is a synthesis telescope, consisting of eight telescopes at three sites in New South Wales. Six of the antennas - comprising the Compact Array - are at the Paul Wild Observatory at Culgoora in north-west New South Wales. Each of the antennas is $22 \mathrm{~m}$ in diameter, and five are movable along a $3-\mathrm{km}$ track. The sixth lies a further $3 \mathrm{~km}$ to the west. The two Telescope are a new 22-m diameter dish near Coonabarabran, and the 27-year-old, 64-m radio telescope at Parkes.

By itself, the Compact Array can map the same fine detail as a telescope $6 \mathrm{~km}$ in diameter. At the $3-\mathrm{cm}$ wavelength it has a
AFTER a decade of planning, design and other antennas making up the Australia resolution of 0.8 seconds of arc - equivalent to being able to read a telephone directory from $200 \mathrm{~m}$ away. When combined with the two other telescopes to make the Long Baseline Array, resolution increases 50 -fold.

The Australia Telescope will be used with telescopes in Australia and in other countries including South Africa, Spain, the Soviet Union and the United States for very long baseline interferometry (VLBI). Australia will also participate in RadioAstron, a space VLBI project of the Soviet Space Agency scheduled for launch in 1992, and the US Quasat (quasar satellite) mission now scheduled for 1996.

The Australia Telescope will open new regions of the radio sky to astronomers. Current Northern Hemisphere telescopes are unable to look further than $37^{\circ} \mathrm{S}$, providing only a limited view of the the centre of our Galaxy.

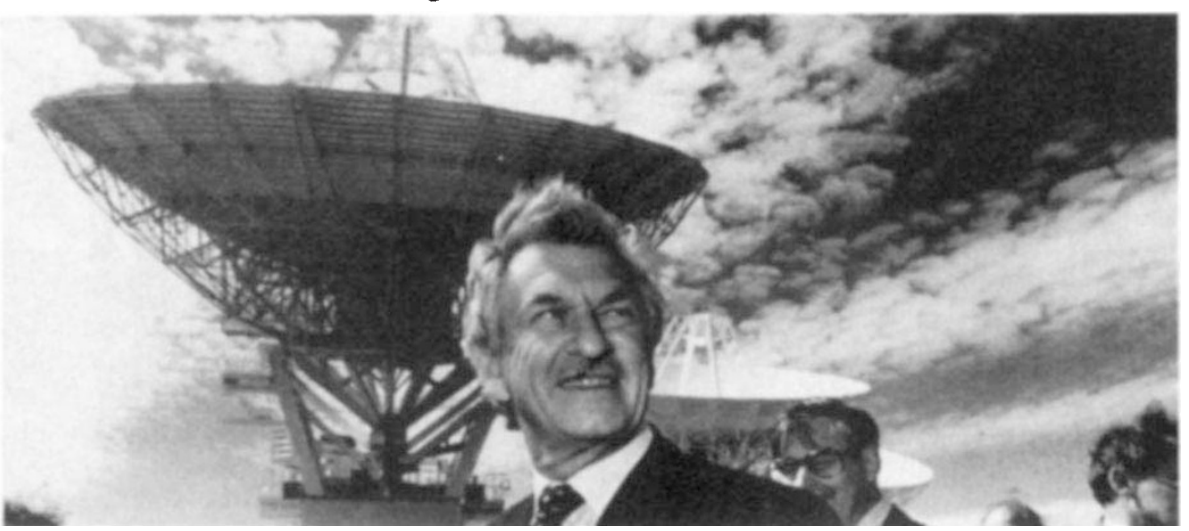

Prime minister Hawke opens the Australia Telescope (AP).

\section{US astronomers gain access to database}

\section{Paris}

A NEw agreement between the French national information-technology and automation research institute (INRIA), in Nice, and two US government agencies, the National Science Foundation (NSF) and the National Aeronautics and Space Administration (NASA) will make it easier for US astronomers to gain access to a key astronomy database in Strasbourg. Until now, computer incompatibility and high costs have made this resource inaccessible to all but a few US astronomers.

The database, SIMBAD (set of identifications, measurements and bibliography for astronomers), enables astronomers to look up an astronomical object by its astronomical designation, gaining access to nearly all known information and a listing of papers about the object since 1950 . But the US Department of Defense standard communications protocol, TCPIP, widely used in American networks, is not compatible with the $X .25$ protocol used in Europe, making it difficult to exchange facilities across the Atlantic. The high costs of using the database, about $\$ 100$ per connection, was also beyond the reach of most astronomers.

Now, with funding from NSF and NASA, these obstacles have been overcome. NSF has paid for about 75 per cent of link between Princeton University and INRIA's Nice campus, with NASA financing the rest, as well as underwriting the charges for use of the database itself. Meanwhile, INRIA has developed a gatetranslate US and European protocols.

After some initial teething troubles when the link was demonstrated in early August, the system is now said by an INRIA spokesman to be fully functional. Peter Coles the costs of a permanent satellite network way (dedicated computer and software) to
The Commonwealth Scientific and Industrial Research Organization (CSIRO) Division of Radiophysics and private industry built the new telescope at a cost of $A \$ 50$ million. It will have an annual operating budget of $\mathrm{A} \$ 5$ million. Dr Ronald Ekers, recently returned to Australia from the Very Large Array at Socorro, New Mexico, in the United States, will be the director.

"The Australia Telescope has a high dynamic range and a wide field of view", says Ekers, "as well as high angular resolution and sensitivity. Australia has now become one of the leaders in world astronomical research."

Tania Ewing

\section{West Germany drills deeper}

Munich

WHY are West German researchers drilling a 14-km-deep hole in Bavaria? Are they seeking oil? Or gas? Not at all, explains Dieter Renz of the Research \& Technology Ministry (BMFT). They simply want to see what there is to see. The BMFT-sponsored project is one of several 'super-deep drilling' projects around the world that will shed light on the structure of the Earth's crust.

Renz has just returned from an international conference at Jaroslavl in the Soviet Union where the Soviets revealed plans to drill 11 holes to depths ranging from 8,000 to $15,000 \mathrm{~m}$. The occasion was only the second at which Westerners had been invited to visit a Soviet deep-drilling rig, this time at Kiluoi Rog near the Black Sea.

In the United States, a hole is being drilled near Cajon Pass in California near the San Andreas fault and has reached a depth of of $5,000 \mathrm{~m}$.

West German researchers successfully reached a depth of $2,970 \mathrm{~m}$ on 1 September with a pilot borehole at Windischeschenbach in Bavaria. They will drill down to $5,000 \mathrm{~m}$ using the same narrow bore, regularly extracting core samples, in preparation for drilling of the main hole, which will begin in late 1989 .

Researchers hope the hole will tell them more about the mechanics of rock formation at depth. The pilot borehole has already revealed interesting results, according to Renz. The rock exposed so far is crystalline, composed primarily of gneiss with varying sillimanite, kyanite and muscovite content. The graphite content is surprisingly high, accounting for the high level of electrical conductivity observed from the surface.

The pilot hole is being drilled using a diamond-tipped bore six inches in diameter lubricated with a viscous silicate compound. Almost all the rock is recovered and analysed. Steven Dickman 